\title{
An experiment to search for mu-e conversion at J-PARC MLF in Japan, DeeMe experiment
}

\author{
Hiroaki Natori* on behalf of DeeMe Collaboration \\ $I B S / C A P P$ \\ E-mail: hatoridibs.re.kp
}

\begin{abstract}
Charged lepton flavor violation (CLFV) is a good probe for new physics beyond the standard model of elementary particle physics. The DeeMe experiment is going to search for one of the CLFV processes, mu-e conversion in a nuclear field, with a sensitivity of $10^{-14}$, ten times better than current limits. The experiment utilizes a fast-extracted proton beam from a Rapid Cycling Synchrotron (RCS) in J-PARC and a production target in Materials and Life Science Experimental Facility (MLF) as a material that muonic atoms are formed. The overview of the experiment and the current status is described.
\end{abstract}

ICHEP 2018, International Conference on High Energy Physics

4-11 July 2018

COEX, Seoul

${ }^{*}$ Speaker. 


\section{Introduction}

When a negative muon stops in a material, the muon is trapped by a nucleus and a muonic atom is formed. Decay in orbit (DIO), $\mu^{-} \rightarrow e^{-}+v_{\mu}+\overline{v_{e}}$, or muon capture, $\mu^{-}+(\mathrm{A}, \mathrm{Z}) \rightarrow$ $v_{\mu}+(\mathrm{A}, \mathrm{Z}-1)$, will occur as major processes. The energy spectrum of electrons from muonic atoms extends higher than half the muon mass due to nuclear recoil. At the endpoint energy of the $\mathrm{DIO}$, monochromatic electrons generated by mu-e conversion, $\mu^{-}+(\mathrm{A}, \mathrm{Z}) \rightarrow e^{-}+(\mathrm{A}, \mathrm{Z})$, may be observed. This is expected as a clear evidence of new physics beyond the Standard Model of elementary particle physics (SM) because the SM predicts charged lepton flavor violation (CLFV) is out of experimental reach, while some models beyond the SM predict large enhancement on the probabilities of the CLFV processes [四]. Many experiments have been performed to search for such events, but none has been observed yet. The current upper limits on the branching ratio of mu-e conversion in nuclei are $7 \times 10^{-13}$ [[]] set by SINDRUM-II experiment for gold, $4.3 \times 10^{-12}$ [B] and $4.6 \times 10^{-12}$ [四] by SINDRUM-II and an experiment at TRIUMF, respectively, for titanium.

\section{DeeMe experiment}

The SINDRUM-II experiment was conducted at PSI using its quasi-continuous muon beam, and a background event was observed near the signal region. It is expected to be a beam related prompt timing background event, so we take a strategy of using a pulsed proton beam and delayed signal time window to get rid of the prompt backgrounds. The DeeMe experiment is schematically shown in Figure W. J-PARC Materials and Life Science Experimental Facility (MLF) delivers the

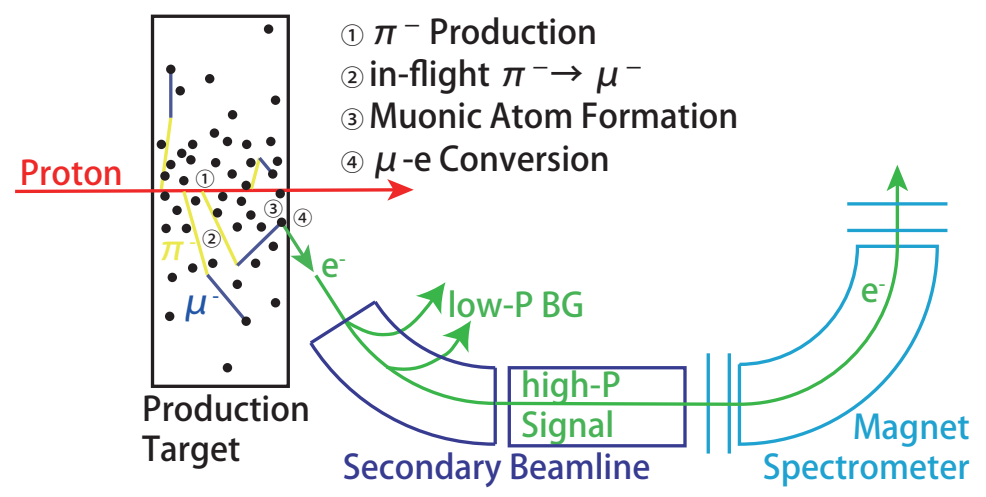

Figure 1: Schematic of the DeeMe experiment

world's most intense pulsed muon beam using the $3 \mathrm{GeV}$ pulsed proton beam with $25 \mathrm{~Hz}$ repetition. A graphite target is equipped in the MLF as a production target to deliver muon beam to secondary beamlines. H-line, a new beamline with a large acceptance, is under construction. DeeMe plans to start the experiment at the $\mathrm{H}$-line utilizing the equipped graphite target as a muonic atom formation material. A series of processes - pion production, pion decay into muon, muon stopping and muonic atom formation - is expected to take place inside the target material and mu-e conversion in muonic carbon may emit approximately $105 \mathrm{MeV}$ electrons with a delay time of $2.0 \mu \mathrm{s}$, the lifetime of muonic carbon. We extract electrons with energy around $105 \mathrm{MeV}$ with the secondary beamline to our detector and measure their momentum and arrival time. The single event sensitivity with 
1 year of data taking $\left(2 \times 10^{7} \mathrm{~s}\right)$ with $1 \mathrm{MW}$ primary proton beam operation is expected to be $8 \times 10^{-14}$. We are also studying the possibility of replacing the target with that made from $\mathrm{SiC}$ which is expected to improve the sensitivity down to $2 \times 10^{-14}$ with 1 year data.

\section{HV switching MWPC}

Proton hits on the production target produce a lot of electrons in the prompt timing. The detector is required to tolerate the prompt burst particles and to detect delayed electrons. Instantaneous hit rate on the detector is expected to be approximately $70 \mathrm{GHz} / \mathrm{mm}^{2}$ at most at the beam center under the condition of $1 \mathrm{MW}$ primary proton beam operation on a $\mathrm{SiC}$ target. To achieve this requirement, we developed a high voltage switching multi-wire proportional chamber (MWPC) []]. We adopted a MWPC composed of anode and potential wires placed alternately in a plane between cathodes as illustrated in Figure $\square$. We switch the high voltage of potential wires on and off as shown in Figure [3]. Initial electrons produced by the prompt burst hits are swept to either anode or potential wires under very suppressed gain condition. This makes it possible to detect delayed signal without suffering from space charge effect. Hit information is read out from cathode strips via amplifiers with large current tolerance [6] and 10-bit flash analog-to-digital converters (FADCs) with a sampling rate of $100 \mathrm{MHz}$ and data compression in field-programmable gate array (FPGA) [四].

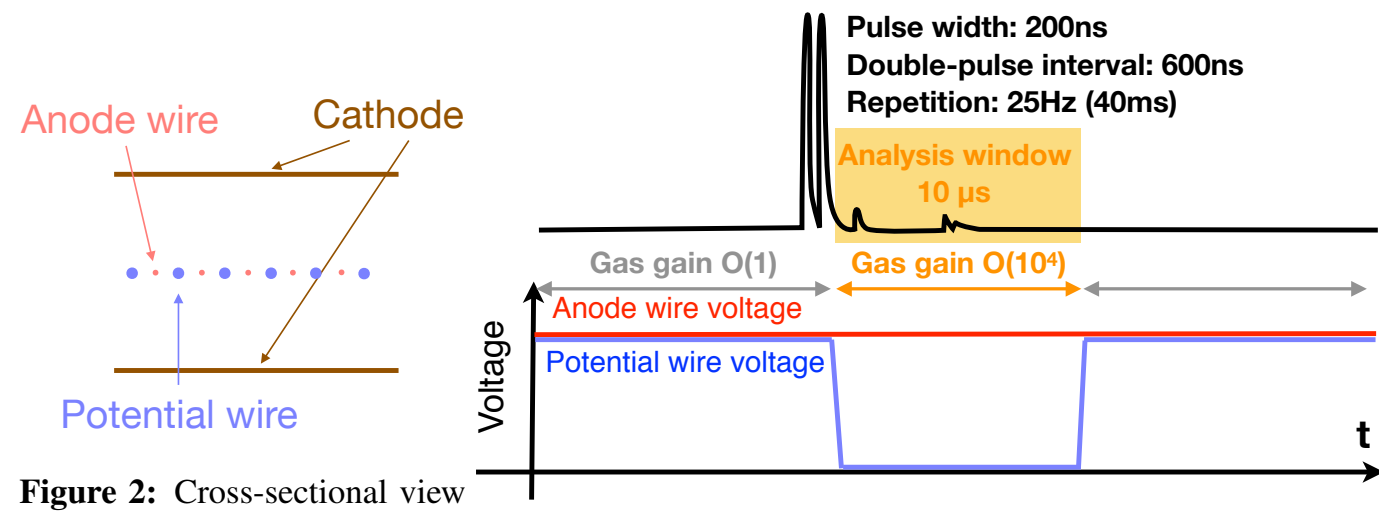
of the MWPC.

Figure 3: Timing schematics of expected electrons at the MWPC and applied voltage on the detector.

\section{Current status}

$\mathrm{H}$-line, the new beamline with a large acceptance, is under construction. Figure $\mathbb{A}$ shows that radiation shields are installed up to a beam area where the DeeMe experiment will be performed.

Figure $\square$ shows a magnet for the spectrometer which was used by PIENU experiment [[] at TRIUMF until 2012 and transported to J-PARC afterwards. We checked its operation and performed magnetic field measurements.

All the 4 chambers are constructed and we performed DIO spectrum measurement around $60 \mathrm{MeV} / \mathrm{c}$ region in D-line, another beamline in MLF. Because the momentum region in scope for 
this measurement was lower and the D2 area is small to install our magnet, a different magnet was used. Several days of data taking was successfully done with the high voltage switching technique.

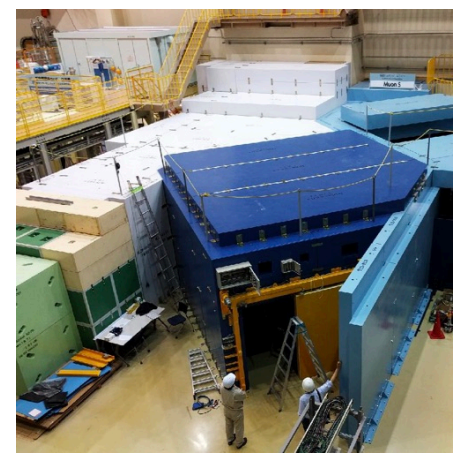

Figure 4: H-line under construction.

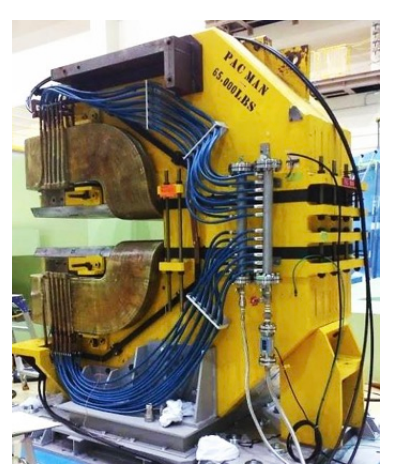

Figure 5: Spectrometer magnet.

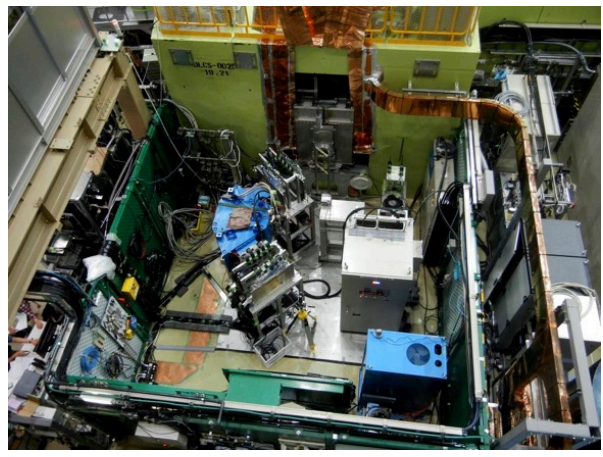

Figure 6: DIO measurement in D2 area.

\section{Summary}

DeeMe plans to search for mu-e conversion in a nuclear field with a sensitivity of $10^{-14}$, ten times better than current limits. The discovery is expected to be a clear evidence for new physics beyond the SM. The detectors and the magnet are tested to be operational. The experiment will start soon after completing the new beamline construction.

\section{References}

[1] A. de Gouvêa and P. Vogel, Prog. Part. Nucl. Phys. 71, 75 (2013).

[2] W. Burtl et al., Eur. Phys. J. C 47, 337 (2006)

[3] C. Dohmen et al., Phys. Lett. B 317, 631 (1993)

[4] S. Ahmad et al., Phys. Rev. D 38, 2102 (1988)

[5] H. Natori et al., Prog. Theor. Exp. Phys. 2017 (2017) 023C01

[6] N. Teshima, in proceedings of FPCP conference PoS(FPCP2015)061.

[7] N. M. Truong et al., IEEE Trans. Nucl. Sci. 65 (2018), 2650

[8] A. Aguilar-Arevalo, et al., Phys. Rev. Lett. 115, 071601 (2015) 